\title{
Длины усиления спектральных компонент собственного стимулированного пикосекундного излучения, зависимость от них характерного времени релаксации указанных компонент и связь спектров стимулированного и спонтанного излучений в GaAs
}

\author{
( Н.Н. Агеева, И.Л. Броневой , Д.Н. Забегаев, А.Н. Кривоносов \\ Институт радиотехники и электроники им. В.А. Котельникова Российской академии наук, \\ 125009 Москва, Россия \\ ฯE-mail: bil@cplire.ru
}

Поступила в Редакцию 28 сентября 2020 г.

В окончательной редакции 10 октября 2020 г.

Принята к публикации 10 октября 2020 г.

\begin{abstract}
При мощной пикосекундной оптической накачке тонкого слоя GaAs в нем образуется интенсивное пикосекундное стимулированное излучение. Спектр излучения представляет собой световой континуум. На базе результатов предыдущих экспериментальных работ сделано следующее. Получены оценки: (а) длин усиления спектральных компонент излучения, т.е. различающихся между собой расстояний, на протяжении которых в активной среде, созданной накачкой, усиливаются различные компоненты излучения; (б) зависимости характерного времени релаксации компонент излучения от их длин усиления. Показано, что спектр произведения длины усиления на коэффициент усиления связывает линейно спектры спонтанного и стимулированного излучений GaAs. Эта связь установлена при насыщении усиления, о котором свидетельствует провал в спектре усиления, „выжженный“ интенсивным излучением.
\end{abstract}

Ключевые слова: стимулированное пикосекундное излучение, спектральные компоненты излучения, длина усиления, характерное время релаксации, насыщение усиления, связь стимулированного и спонтанного излучений, арсенид галлия.

DOI: 10.21883/FTP.2021.02.50496.9526

\section{1. Введение}

В настоящей работе описывается основывающаяся на экспериментальных данных оценка длин усиления $L$ спектральных компонент собственного стимулированного интенсивного пикосекундного излучения GaAs (далее, для краткости, оно называется излучение). Излучение возникало при пикосекундной оптической накачке тонкого слоя GaAs. Этот слой входил в состав гетероструктуры $\mathrm{Al}_{0.22} \mathrm{Ga}_{0.78} \mathrm{As}-\mathrm{GaAs}-\mathrm{Al}_{0.4} \mathrm{Ga}_{0.6} \mathrm{As}$. Представление об изменении со временем $t$ интенсивности излучения $I_{\Sigma}$, интегрального по спектру, представлено графиком, называемым хронограммой, на рис. 1. Оценивается длина усиления $L$, средняя за время излучения. Полученный в итоге спектр длин усиления $L(\hbar \omega)$ вместе с измеренным ранее спектром усиления света $\alpha(\hbar \omega)$ дают простую связь форм спектров спонтанного и стимулированного излучений GaAs. Он также позволил сделать оценку зависимости характерного времени релаксации спектральных компонент излучения от длины их усиления. Аналогичная зависимость характерного времени релаксации излучения, но только интегрального по спектру, и в функции от диаметpa $(F W H M)$ луча накачки была обнаружена и объяснена в работе [1]. При описываемых далее оценках мы использовали результаты наших измерений в предыдущих работах. Они не всегда проводились в абсолютно одинаковых условиях, что делает наш вывод прибли- женным. Но некоторое различие в накачке близких по структуре образцов не влияло на очевидность основных выводов.

\section{2. К чему ведет пространственная неоднородность накачки}

Обсудим сначала, как возникает и как должна выглядеть зависимость длины усиления компоненты излучения от энергии ее фотона. При пространственно неоднородном, например, гауссовом распределении интенсивности света накачки по сечению ее луча длины усиления различных спектральных компонент излучения должны быть различны. Это схематически поясняет рис. 2. Он несколько сходен с рис. 1 в работе [3], в которой предполагалось, что неоднородное пространственное распределение накачанных носителей возникает из-за пространственно неоднородного распределения интенсивности излучения при насыщении его усиления и стационарной накачке. На рис. 2 кривая 1 отображает фрагмент близкого к гауссову распределения плотности неравновесных носителей заряда $n=p$ вдоль диаметра накачанной области GaAs. B термодинамически неравновесном состоянии этой области единого уровня Ферми для нее уже не существует, вместо него вводятся квазиуровни Ферми для электронов $\mu_{\mathrm{e}}$ и для дырок $\mu_{\mathrm{h}}$. С появлением неравновесных носителей в зоне проводимости 


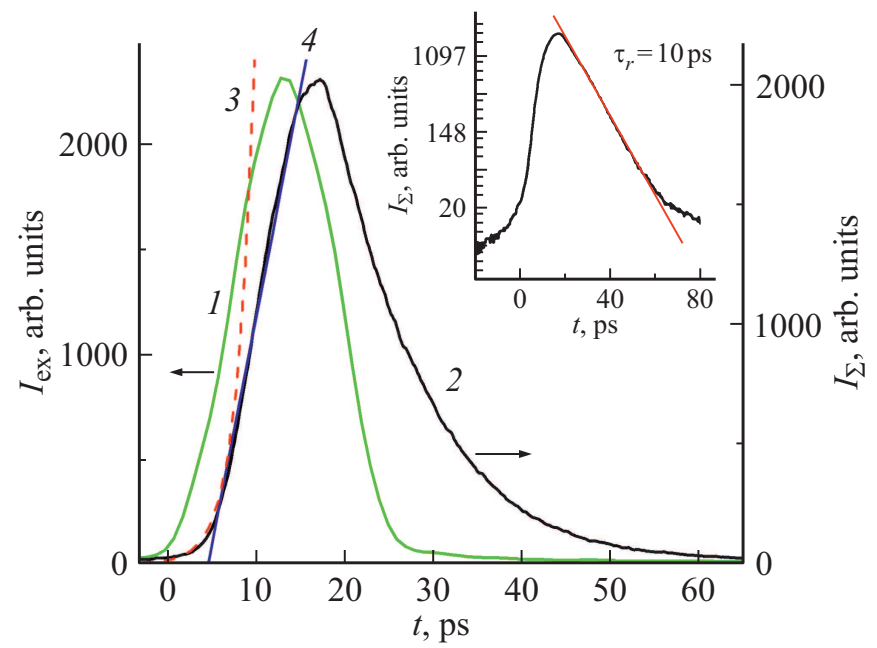

Рис. 1. Хронограммы импульса накачки $I_{\mathrm{ex}}(t)(1)$ и излучения $I_{\Sigma}(t)(2)$, интегрального по спектру [2]. Кривые (3), (4) поясняются в тексте. На вставке показана хронограмма излучения $I_{\Sigma}(t)$ в полулогарифмическом масштабе. К спаду хронограммы проведена касательная, справа от которой указано характерное время $\tau_{r}$ релаксации интенсивности излучения.
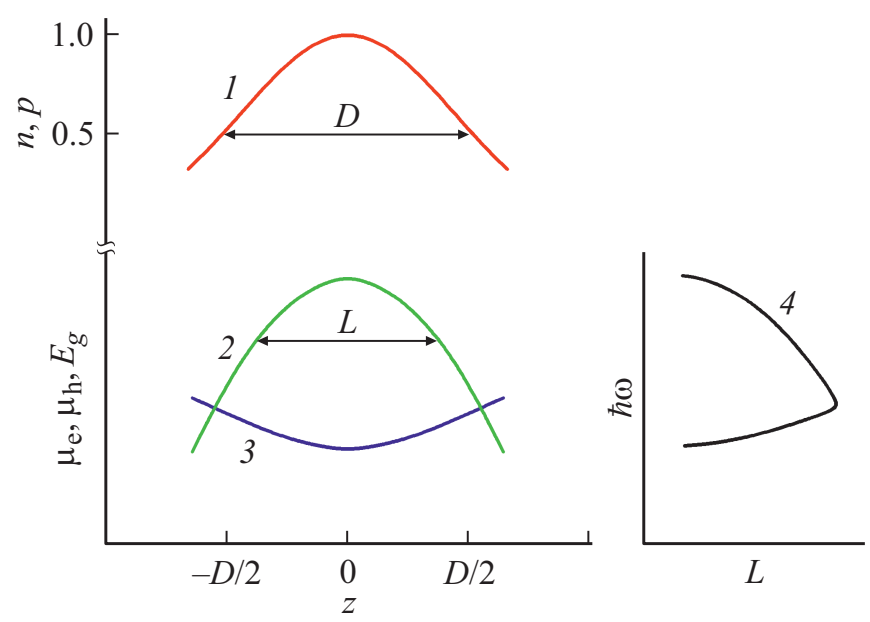

Рис. 2. Схематическое представление распределения в пространстве: плотности носителей заряда (1), разности квазиуровней Ферми $\left(\mu_{\mathrm{e}}-\mu_{\mathrm{h}}\right)(2)$, ширины запрещенной зоны $E_{g}(3)$, ожидаемых при гауссовом распределении интенсивности света накачки по сечению ее луча, здесь $z-$ координата точки, лежащей на диаметре накачанной области $\mathrm{GaAs}, D-$ диаметр луча накачки. Кривая 4 - изменение длины усиления $L$ спектральной компоненты излучения при изменении энергии ее фотона $\hbar \omega$.

и в валентной зоне происходит как бы „расщепление“ первоначального уровня Ферми на два квазиуровня $\mu_{\mathrm{e}}$ и $\mu_{\mathrm{h}}$, каждый из которых смещается по направлению к своей зоне по мере возрастания плотности носителей [4]. Зависимость $\mu_{\mathrm{e}}-\mu_{\mathrm{h}}=f(z)$ отображает кривая 2 на рис. 2, где $z$ - координата точки, лежащей на диаметре накачанной области GaAs.
Когда при накачке разность квазиуровней Ферми увеличится настолько, что превысит ширину запрещенной зоны $E_{g}$, то создастся инверсия населенности электронами таких энергетических уровней зоны проводимости, при рекомбинации с которых электроны излучают фотоны спектрального диапазона [5]:

$$
\mu_{\mathrm{e}}-\mu_{\mathrm{h}} \geq \hbar \omega \geq E_{g} .
$$

Имеется в виду инверсия относительно заселенности электронами уровней в области вершины валентной зоны, связанных прямыми оптическими переходами с указанными уровнями зоны проводимости. Свет с энергией фотонов, соответствующей (1), будет усиливаться. Когда усиление излучения при распространении вдоль слоя GaAs превысит потери, возникнет стимулированное излучение. Для примера к соотношению (1) приведем оценки $\mu_{\mathrm{e}}=0.104$ эВ $E_{g}+\mu_{\mathrm{h}}=0.066$ эВ, сделанные в работе [6] из измеренного спектра просветления (увеличения прозрачности, вызванного накачкой носителей) слоя GaAs, уровни Ферми отсчитаны от дна зоны проводимости.

Из-за кулоновского взаимодействия носителей происходит перенормировка (сужение) запрещенной зоны [7]:

$$
\Delta E_{g} \sim n^{1 / 3} .
$$

Перенормировка запрещенной зоны наблюдалась в ряде наших работ, в частности в статье [8]. С учетом перенормировки зависимость $E_{g}(z)$ выглядит подобно кривой 3 на рис. 2. Замкнутая область, ограничиваемая кривыми 2 и 3 , удовлетворяет условию (1) и позволяет представить, как меняется длина усиления $L$ спектральной компоненты излучения при изменении энергии ее фотона $\hbar \omega$. Coответствующую форму зависимости $L(\hbar \omega)$ схематично иллюстрирует кривая 4 на рис. 2. Упрощенно ее можно считать подобной асимметричному колоколу.

\section{3. Связь спектров стимулированного и спонтанного излучений и спектр длин усиления}

Перейдем к определению спектра длин усиления $L(\hbar \omega)$. На рис. 3 показаны интегральный по времени спектр стимулированного излучения $W_{s 1}(\hbar \omega)$ (кривая 1$)$ и спектр коэффициента усиления зондирующего импульса света $\alpha(\hbar \omega)$ (кривая 3) задержанного относительно накачки на 3 пс. Эти спектры, полученные экспериментально, взяты из работы [9]. На том же рисунке приведен экспериментальный спектр спонтанного излучения $W_{s p}(\hbar \omega)$, интегральный по времени (кривая 2). Измерялась часть спонтанного излучения, выходившего приблизительно ортогонально плоскости эпитаксиальных слоев настолько совершенной гетероструктуры, что рассеиваемая в том же направлении часть стимулированного излучения была много меньше. Указанный выше спектр $W_{s 1}(\hbar \omega)$ в действительности является суммой 


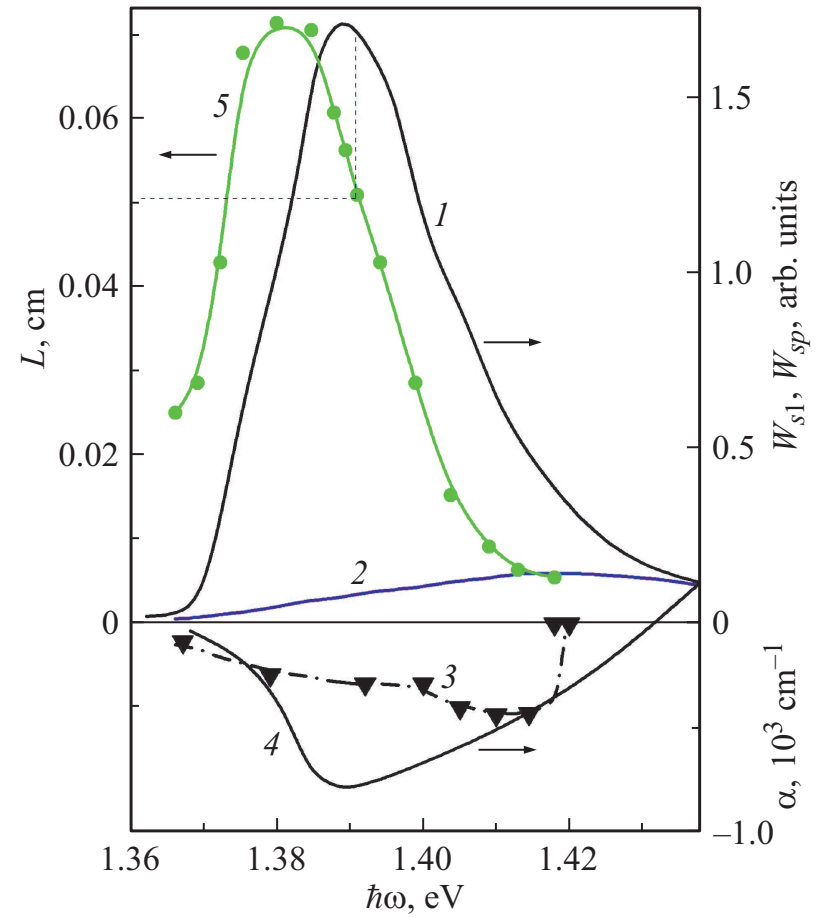

Pис. 3. Интегральные по времени спектры стимулированного излучения $W_{s 1}(\hbar \omega)(1)$ и спонтанного излучения $W_{s p}(\hbar \omega)(2)$. Область усиления в спектре поглощения зондирующего импульса света, отстававшего на 3 пс от импульса накачки, $\alpha(\hbar \omega)$ (3). Расчетный спектр поглощения при фермиевском распределении ЭДП (4). Спектр длины усиления $L$ (5).

спектров стимулированного и спонтанного излучений. В том спектральном диапазоне, где усиление излучения значительно, вкладом спонтанного излучения пренебрегалось из-за его малости по сравнению со стимулированным излучением. Но та коротковолновая часть спектра $W_{s 1}(\hbar \omega)$, где усиления нет, а есть не показанное на рисунке поглощение света, представляет собой спонтанное излучение. Таким образом, в этой „коротковолновой“ спектральной области спектры $W_{s 1}(\hbar \omega)$ и $W_{s p}(\hbar \omega)$ должны быть одинаковы. Чтобы это выполнялось, спектр $W_{s p}(\hbar \omega)$, измеренный в другой работе, в отличие от спектра $W_{s 1}(\hbar \omega)$, был соответственно отнормирован. В том, что на рис. $3 \alpha=0$ при $\hbar \omega=1.42$ эВ, а спектры еще немного отличаются при $\hbar \omega \leq 1.434$ эВ, нет противоречия. Дело в том, что вблизи последней энергии фотона была расположена коротковолновая граница спектра стимулированного излучения, который со временем из-за перенормировки $E_{g}$ сдвигался в длинноволновую сторону [2]. Во время измерения спектра $\alpha(\hbar \omega)$, проиллюстрированного на рис. 3 , усиление излучения с энергией фотона 1.42 эВ $<\hbar \omega<1.434$ эВ было уже за пределами чувствительности наших измерений.

Экспериментальный спектр усиления отличается „выжженным“ излучением провалом от того расчетного спектра, (кривая 4 на рис. 3), какой бы был при фермиевском распределении носителей заряда. Наличие провала говорит о насыщении усиления, в условиях которого излучение должно усиливаться не экспоненциально, а линейно согласно $[10,11]$. Этому отвечает, видимо, и то, что на фронте интенсивность излучения только в самом начале до $t \approx 7$ пс растет экспоненциально, как показывает график экспоненты 3 на рис. 1, а затем интенсивность увеличивается со временем линейно, что подтверждает касательная прямая 4 на том же рисунке. В согласии с этим и с данными исследования провала из работы [9] очевидно, что насыщение усиления имеет место на протяжении большей части времени излучения, и поэтому можно принять, что приближенно при $\hbar \omega<1.42$ эВ выполняется соотношение

$$
W_{s 1} \approx-\alpha \cdot L \cdot W_{s p}
$$

Получаемый с помощью (3) спектр длины усиления

$$
L \approx W_{s 1} /\left(-\alpha \cdot W_{s p}\right)=f(\hbar \omega)
$$

представлен на рис. 3 кривой 5 . График спектра $L(\hbar \omega)$ имеет форму, как и предполагалось выше, несимметричного колокола.

Ранее в работе [12] было установлено и повторяется далее в разд. 4 следующее. Спектральная компонента, длина усиления которой равна диаметру $D$, расположена в области максимума спектра стимулированного излучения. Как показано пунктиром на рис. 3, подобное справедливо для полученного спектра длин усиления. Этим дополнительно подтверждается его реалистичность.

\section{4. Зависимость характерного времени релаксации компонент излучения от длины их усиления}

Теперь открывается возможность установить зависимость характерного времени релаксации компонент излучения $\tau_{r-m}$ от длины усиления компонент. Сделаем это для излучения, исследоваванного в работе [2]. Время $\tau_{r-m}$ было определено из хронограмм компонент, измеренных в этой работе, и получившийся спектр $\tau_{r-m}(\hbar \omega)$ представлен кривой 4 на рис. 4. Спектр длин усиления был получен следующим образом. Как подтверждает рис. 4, формы спектров стимулированного излучения, измеренных в работе [9], $-W_{s 1}(\hbar \omega)$, кривая 2, верхняя ось абсцисс, и в работе [2] $W_{s 2}(\hbar \omega)$, кривая 1 , нижняя ось абсцисс, практически совпадают, хотя они сдвинуты один относительно другого на 12 мэВ. Сдвиг вызван, видимо, различием ширины запрещенной зоны $E_{g}$, вызванным, скорее всего, разной напряженностью кристаллов $\mathrm{GaAs}$ или др. Естественно предполагать, что спектры длины усиления в этих работах тоже имеют одинаковую форму и одинаково спектрально расположены по отношению к указанным спектрам стимулированного излучения, имеющим одинаковую форму. В соответствии с этим мы получаем 


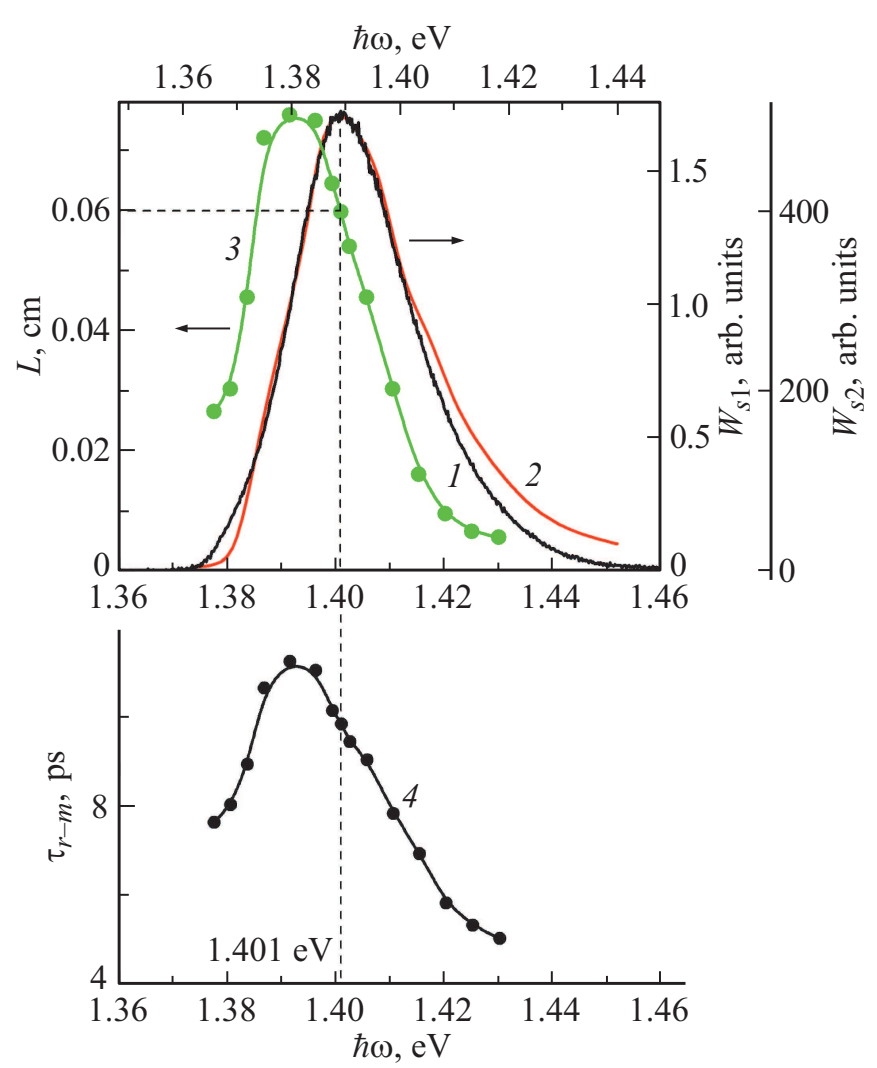

Рис. 4. Интегральные по времени спектры стимулированного излучения $W_{s 1}(\hbar \omega)(1)$ из работы [2] нижняя ось абсцисс и $W_{s 2}(\hbar \omega)(2)$ верхняя ось абсцисс из работы [9]. Спектр длины усиления $L(\hbar \omega)(3)$. Зависимость характерного времени $\tau_{r-m}$ релаксации интенсивности спектральных компонент излучения от энергии их фотона (4).

спектр $L(\hbar \omega)$ для данных из работы [2], представленный на рис. 4 графиком 3, относящимся к нижней оси абсцисс. Выражение $L$ в абсолютных единицах длины, как представлено на рис. 4, было получено, используя поясняемую далее взаимосвязь диаметра активной области $D$ с характерным временем релаксации излучения.

В работе [13] было получено аналитическое выражение характерного времени $\tau_{T}$ остывания оптически накачанных носителей заряда в GaAs. Остывание происходило в присутствии излучения GaAs, которое создавало дополнительный нагрев носителей заряда, увеличивавший $\tau_{T}$. В работе [1] было показано, что благодаря взаимосвязи плотности и температуры неравновесных носителей заряда, возникающей при интенсивном излучении $\mathrm{GaAs}$, время $\tau_{T}$ и характерное время $\tau_{r}$ релаксации интенсивности излучения становятся взаимосвязаны. Отсюда следовало выражение для времени $\tau_{r}$, доступного для прямых измерений:

$$
\tau_{r} \approx(2 / 3) \tau_{T} \approx(2 / 3)\left(A+B \tau_{p} T_{c}^{1 / 2} E_{g}\right) \tau_{h},
$$

где $A=6.3, B=0.36, \tau_{h} \approx 0.8$ пс - время релаксации энергии электронно-дырочной плазмы (ЭДП) за счет эмиссии оптических фононов с учетом разогрева последних [14], $\tau_{r}, \tau_{p}, \tau_{h}$ измеряются в пс, $T_{c}-$ в К, $E_{g}$ - в эВ. Первое слагаемое в выражении (5) учитывает разогрев ЭДП, связанный с тем, что энергия носителей заряда, участвующих в стимулированной рекомбинации, меньше средней энергии носителей в ЭДП. Второе слагаемое учитывает разогрев ЭДП из-за внутризонного поглощения собственного излучения. Время $\tau_{p}-$ это характерное время движения фотона излучения в активной среде, пока фотон не покинет эту среду или не будет поглощен:

$$
\tau_{p}^{-1} \approx c \cdot \chi^{-1}\left(\gamma+D^{-1}\right) .
$$

Здесь $\chi=3.6$ - показатель преломления $\mathrm{GaAs}$, $\gamma=\sigma n-$ коэффициент внутризонного поглощения света, $\sigma=1.5 \cdot 10^{-17} \mathrm{~cm}^{2}$ [15]. Следовавшая из (6) и (7) взаимосвязь времени $\tau_{r}$ и диаметра $D$ удовлетворительно согласовалась с измеренной в эксперименте [1] зависимостью $\tau_{r}(D)$, представленной кривой 1 на рис. 5 . Для этого графика диаметр активной области считался равным диаметру сечения луча накачки на полувысоте $D(F W H M)$, а $\tau_{r}$ являлось характерным временем релаксации интенсивности излучения, интегрального по спектру. На вставке в рис. 1 зависимость $I_{\Sigma}(t)$ показана в полулогарифмическом масштабе, и к ее спаду проведена касательная линия, показывающая, что релаксацию излучения можно аппроксимировать как экспоненциальную с характерным временем $\tau_{r}$, указанным рядом с касательной. В работе [12] отмечалось, что время $\tau_{r}$ совпадало с характерным временем релаксации $\tau_{r-m}$ той спектральной компоненты излучения, интенсивность которой превышала на спаде излучения интенсивность остальных компонент. Для работы [2] подобную роль выполняла компонента с $\hbar \omega=1.401$ эВ, хронограмма которой показана кривой 2 на рис. 6 в сравнении с

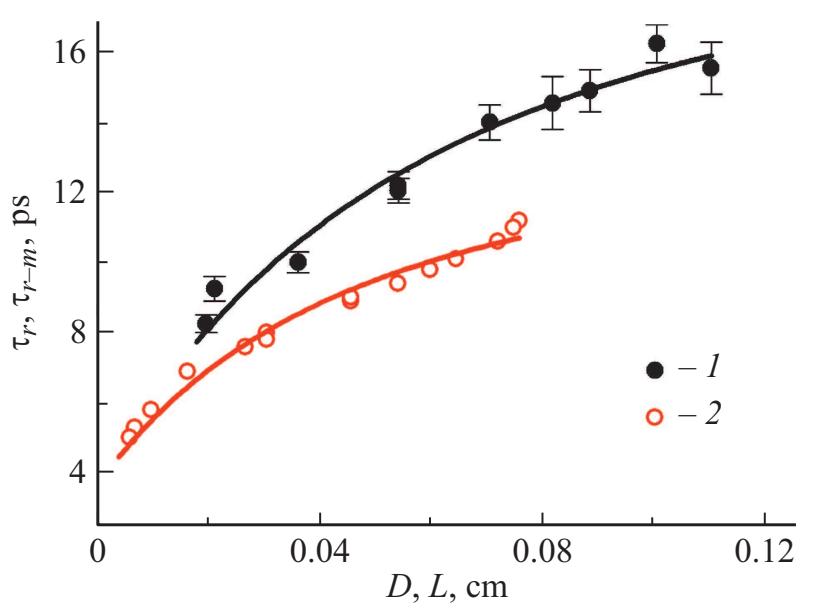

Рис. 5. Зависимости характерного времени $\tau_{r}$ релаксации интенсивности интегрального по спектру излучения от диаметра $D(1)$ (из работы [1]) и характерного времени $\tau_{r-m}$ релаксации интенсивности спектральных компонент излучения от длины усиления $L(2)$. Сплошные линии проведены для наглядности. 


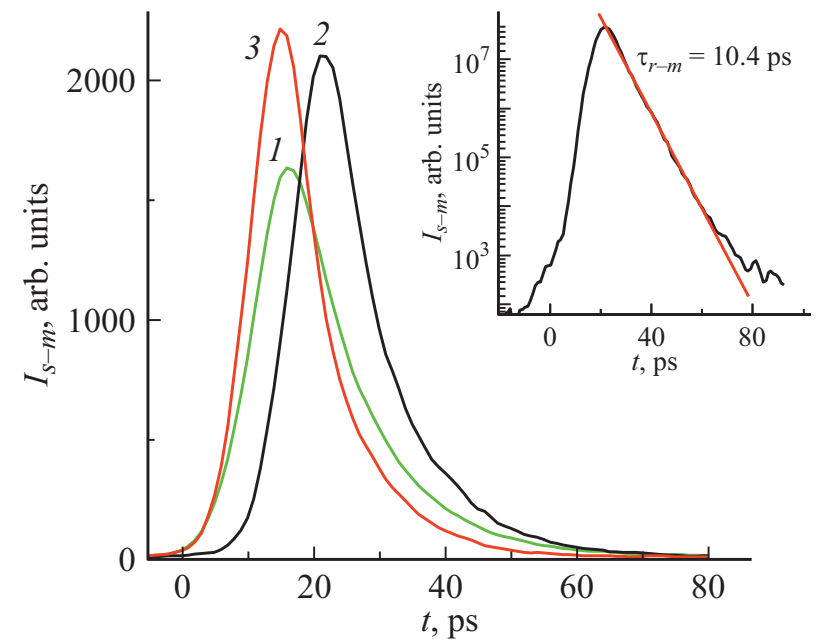

Рис. 6. Хронограммы избранных спектральных компонент $I_{s-m}(t)$ излучения с энергиями фотона $\hbar \omega$, эВ: $1-1.396$, $2-1.401,3-1.406$. На вставке показана хронограмма компоненты излучения с $\hbar \omega=1.401$ эВ в полулогарифмическом масштабе. К спаду хронограммы проведена касательная, справа от которой указано характерное время $\tau_{r-m}$ релаксации интенсивности излучения.

хронограммами еще двух компонент. Для компоненты с $\hbar \omega=1.401$ эВ практически выполняется равенство $\tau_{r-m} \approx \tau_{r}$, что видно из сопоставления вставок на рис. 1 и 6. Но при таком равенстве, как того требуют (5) и (6), длина усиления этой компоненты равна диаметру луча накачки, т.е. при $\hbar \omega=1.401$ эВ, $L \approx D=0.06$ см. Приравнивая этому значению $L$ при $\hbar \omega=1.401$ эВ на спектре $L(\hbar \omega)$ для данных из работы [2], как показано пунктиром на рис. 4 , мы получаем значения $L$ в сантиметрах для всего спектра (кривая 3 на рис. 4).

Зависимость $\tau_{r-m}(L)$ можно получить из сопоставления полученного графика $L(\hbar \omega)$ с графиком экспериментальной зависимости $\tau_{r-m}(\hbar \omega)$ на рис. 4 . Кривая 2 на рис. 5 представляет получающуюся зависимость $\tau_{r-m}=f(L)$. Как видно, она подобна зависимости $\tau_{r}(D)$ в [1], но график $\tau_{r-m}=f(L)$ расположен несколько ниже, т.е. $\tau_{r-m}<\tau_{r}$ при $D=L$. Это вызвано тем, что из-за более интенсивной накачки плотность носителей в [2] была больше, а время $\tau_{p}$, согласно (2), меньше, чем в [1].

\section{5. Заключение}

В итоге на основании представлений о линейности усиления излучения при насыщении усиления была получена из экспериментальных данных, в пределах их точности, оценка спектра длин усиления спектральных компонент излучения в GaAs в работе [9]. Аналогичная оценка для работы [2] потребовала еще учета связи между временем релаксации излучения и диаметром активной области. Реалистичность этих оценок, как и представления о линейности усиления, подтвердилась дополнительно, во-первых, тем, что спектр длины усиления компонент имел ожидаемую форму асимметричного колокола, и длина усиления компоненты из области максимума спектра энергии излучения совпадала с диаметром $(F W H M)$ луча накачки. Во-вторых, тем, что полученная оценка зависимости характерного времени релаксации спектральных компонент излучения от длины их усиления, имеющая самостоятельную ценность, была близка к зависимости характерного времени релаксации интегрального по спектру излучения от диаметра луча накачки. Нам не известно, чтобы ранее были получены из экспериментальных результатов подобные характеристики стимулированного излучения GaAs.

Подчеркнем важность того, что при полученном спектре длин усиления согласовываются экспериментальные спектры излучения и усиления, соответствовавшего нефермиевскому распределению носителей заряда. Тогда как в монографиях, например, [16,17], рассматривая стимулированное излучение, преимущественно приводят расчетные спектры усиления для фермиевского распределения носителей, которые при интенсивном излучении далеки от реальных.

\section{Финансирование работы}

Работа выполнена в рамках государственного задания.

\section{Конфликт интересов}

Авторы заявляют, что у них нет конфликта интересов.

\section{Список литературы}

[1] Н.Н. Агеева, И.Л. Броневой, Д.Н. Забегаев, А.Н. Кривоносов. ФТП, 53, 1471 (2019).

[2] Н.Н. Агеева, И.Л. Броневой, Д.Н. Забегаев, А.Н. Кривоносов. ЖЭТФ, 144, 227 (2013).

[3] E.O. Goebel, O. Hildebrand, K. Lohnert. IEEE J. Quant. Electron., 13, 848 (1977).

[4] В.Л. Бонч-Бруевич, С.Г. Калашников. Физика полупроводников (М., Наука, 1981).

[5] О. Звелто. Принципы лазеров (СПб., Лань, 2008).

[6] N.N. Ageeva, I.L. Bronevoi, E.G. Dyadyushkin, V.A. Mironov, S.E. Kumekov, V.I. Perel'. Sol. St. Commun., 72, 625 (1989).

[7] J. Shah, R.F. Leheny, C. Lin. Sol. St. Commun., 18, 1035 (1976).

[8] Н.Н. Агеева, И.Л. Броневой, Д.Н. Забегаев, А.Н. Кривоносов. ФТП, 51, 594 (2017).

[9] Н.Н. Агеева, И.Л. Броневой, А.Н. Кривоносов, С.Е. Кумеков, С.В. Стеганцов. ФТП, 36, 144 (2002).

[10] L.W. Casperson. J. Appl. Phys., 48, 256, (1977).

[11] В.Д. Соловьев. Физика лазеров. Текст лекций (4-й курс). http://elib.spbstu.ru/d1/2313.pdf/download/2313.pdf

[12] Н.Н. Агеева, И.Л. Броневой, Д.Н. Забегаев, А.Н. Кривоносов. ФТП, 55 (2), 113 (2021).

[13] Ю.Д. Калафати, В.А. Кокин. ЖЭТФ, 99, 1793 (1991).

[14] С.Е. Кумеков, В.И. Перель. ЖЭТФ, 94, 346 (1988).

[15] J.S. Blakemore. J. Appl. Phys., 53, R123 (1982). 
[16] Х. Кейси, М. Паниш. Лазеры на гетероструктурах (М., Мир, 1981).

[17] Физика полупроводниковых лазеров, под ред. Х. Такумы (М., Мир, 1989).

Редактор А.Н. Смирнов

The amplification lengths of the spectral components of the intrinsic stimulated picosecond emission, the dependence of the characteristic relaxation times of these components on their amplification lengths, the relationship between the spectra of stimulated and spontaneous emissions in GaAs

N.N. Ageeva, I.L. Bronevoi, D.N. Zabegaev, A.N. Krivonosov

Kotel'nikov Institute of Radioengeneering and Electronics, Russian Academy of Sciences, 125009 Moscow, Russia

Abstract High-power picosecond optical pumping of a thin GaAs layer generates intense picosecond stimulated emission. The emission spectrum is a light continuum. Based on the results of previous experimental work, the following was done. Estimates were obtained: (a) the amplification lengths of the spectral components of the emission, i. e. differing distances over which different components of the emission are amplified in the active medium created by the pump; (b) the dependence of the characteristic relaxation time of the emission components on their gain lengths. It is shown that the spectrum of the product of the gain length and the gain coefficient linearly relates the spectra of spontaneous and stimulated emission of GaAs. This connection was established at gain saturation, which is evidenced by a dip in the gain spectrum, „burned out" by intense emission. 